\title{
Power Balance and Temperature in Optically Pumped Spasers and Nanolasers
}

\author{
Gerold V. Kristanz, ${ }^{\dagger}$ Nikita Arnold, ${ }^{* \dagger, \ddagger, \S ® ~ A l e x a n d e r ~ V . ~ K i l d i s h e v, ~ " ~ a n d ~ T h o m a s ~ A . ~ K l a r ~}{ }^{\dagger}$ \\ ${ }^{\dagger}$ Institute of Applied Physics, Johannes Kepler University, Altenberger Straße 69, 4040, Linz, Austria \\ ${ }^{\ddagger}$ Institute of Semiconductor and Solid State Physics, Johannes Kepler University, Altenberger Straße 69, 4040, Linz, Austria \\ "Birck Nanotechnology Center, School of ECE, Purdue University, West Lafayette, 1205 West State Street, Indiana 47907-2057, \\ United States
}

\section{Supporting Information}

ABSTRACT: Spasers and nanolasers produce a significant amount of heat, which impedes their realizability. We numerically investigate the farfield emission and thermal load in optically pumped spasers with a coupled electromagnetic/thermal model, including additional temperature discontinuities due to interfacial Kapitza resistance. This approach allows to explore multiple combinations of constitutive materials suitable for robust manufacturable spasers. Three main channels of heat generation are quantified: metal absorption at pumping and spasing wavelengths and nonradiative relaxations in the gain material. Outradiated power becomes comparable with absorption for

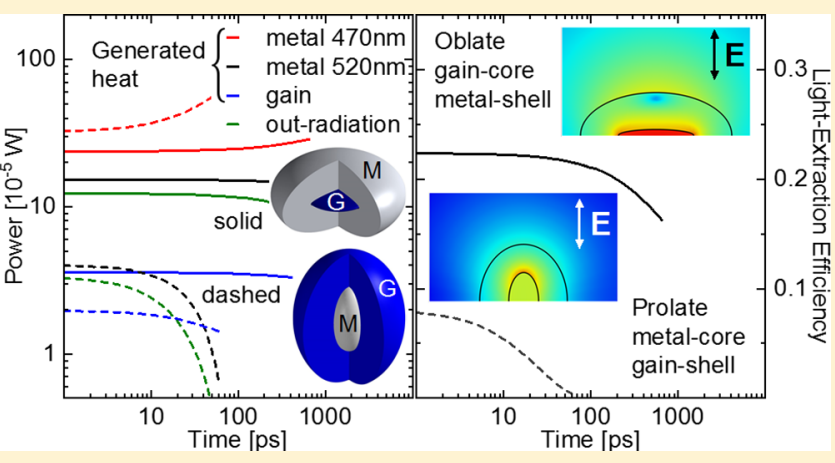
spasers of realistic dimensions. Two optimized spaser configurations emitting light near $520 \mathrm{~nm}$ are compared in detail: a prolate metal-core/gain-shell and an oblate gain-core/ metal-shell. The metal-shell design, which with the increasing size transforms into a metal-clad nanolaser, achieves an internal light-extraction efficiency of $22.4 \%$, and stably operates up to several hundred picoseconds, an order of magnitude longer than the metal-core spaser.

KEYWORDS: plasmonics, spasing, gain saturation, nanoparticle heating, power balance, light extraction efficiency, Kapitza resistance, core-shell spheroids

$\mathrm{O}$ ne of the principal problems of the realization of a spaser (surface plasmon amplifier using stimulated emission) and metal-clad nanolaser is that there will be strong fields in an absorbing metal component and its vicinity. ${ }^{1}$ As metals are very good at converting electromagnetic energy into heat, this increases the temperature of the system. In general, the heating of plasmonic nanostructures is interesting in two different aspects: either the heating is a desired effect, for example, for electrothermoplasmonic nanotweezers in microfluidic channels ${ }^{2}$ or medical applications of nanoparticles in living tissues, ${ }^{3}$ or it threatens the realizability of a device. In lowest order approximation, the surface plasmon oscillations in a spaser decay primarily through ohmic loss. The spasing modes also loose energy through far-field radiation (e.g., when used as a nanoscopic light source). In the quasi-static case, this is a weaker effect, but for the spasers of realistic dimensions, especially for "bright" dipolar modes, this channel may become comparable with absorption and important; it is included in our study. In a realistic setting, a temperature change of several hundred degrees in a fraction of a nanosecond is possible. The thermal behavior of spasers and surface plasmons has been discussed by several groups. For example, Smalley et al. ${ }^{4}$ derived a framework for the temperature dependence of the spontaneous emission in semiconductor nanolasers (primarily photonic, but also plasmonic), and estimative approximations regarding the heating of spasers have been carried out by Fedorov et al. ${ }^{5}$ However, a comprehensive analysis of the thermal behavior of an operating spaser, as presented in the following study, was missing so far. We aim to provide practical guidelines for experimenters primarily on two subjects, suggest realistic geometries for individual spasers and give estimates for pumping intensities and durations, as well as expected outradiation and temperatures. This is the first study combining the effects of spatially dependent gain saturation, retardation and radiative losses (i.e., light extraction), heat generation in both metal and gain material, Kapitza resistance, temperature dependences of material properties, and influence of these factors on the temporal evolution of spasing.

\section{ELECTRODYNAMIC DESCRIPTION OF A SPASER}

Gain Dielectric Function. We use the gain dielectric function derived in ref 6

Received: May 24, 2018

Published: August 24, 2018 


$$
\varepsilon_{\mathrm{G}}=\varepsilon_{\mathrm{h}}-\varepsilon_{\mathrm{L}} \frac{\left(\omega_{21}-\omega_{\mathrm{s}}+i \gamma_{\mathrm{L}} / 2\right)\left(\gamma_{\mathrm{L}} / 2\right)}{\left(\omega_{21}-\omega_{\mathrm{s}}\right)^{2}+\left(\gamma_{\mathrm{L}} / 2\right)^{2}\left(1+\left(\left|\mathbf{E}_{\mathrm{s}}\right| / E_{\mathrm{sat}}\right)^{2}\right)}
$$

which is based on a simplified 4-level scheme for the chromophores (see Figure 1) and accounts for gain saturation

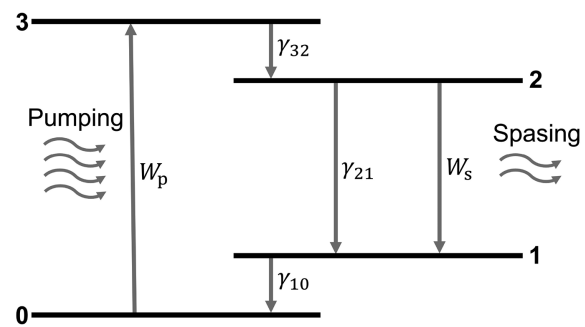

Figure 1. Simplified 4-level scheme used to model the chromophores. The pumping transition is from levels $0 \rightarrow 3$, and the spasing transition is from levels $2 \rightarrow 1$. We assume fast relaxation from levels 3 and $1,\left(\gamma_{32}, \gamma_{10}\right) \gg\left(W_{s}, W_{p}, \gamma_{21}\right)$.

(SI units are used in this work). The underlying assumptions are fast dephasing of the chromophore polarizations $\gamma_{\mathrm{L}} \gg \gamma_{i j}$ and fast relaxation from levels 3 and $1\left(\gamma_{32}, \gamma_{10}\right) \gg\left(W_{\mathrm{s}}, W_{\mathrm{p}}, \gamma_{21}\right)$. Here, $\omega_{\mathrm{s}}$ is the frequency of surface plasmon oscillations (the spasing frequency internally established in the system) and $\varepsilon_{\mathrm{h}}$ is the dielectric function of the gain host material. The emission of the chromophores is described by a Lorentzian with strength $\varepsilon_{\mathrm{L}}$ and width $\gamma_{\mathrm{L}}$, centered at a fixed, central freespace wavelength corresponding to the $2 \rightarrow 1$ molecular transition, $\lambda_{21}=2 \pi c / \omega_{21}$, with $c$ being the vacuum speed of light. The dimensionless amplitude of the emission Lorentzian at midline, $\varepsilon_{\mathrm{L}}$, is called (unsaturated) gain level. It is directly related to the pumping rate $W_{\mathrm{p}}\left[\mathrm{s}^{-1}\right]$ (adapted from eqs 18 and 22 in ref 6),

$$
\varepsilon_{\mathrm{L}}=\frac{c}{\omega_{\mathrm{s}}} \sqrt{\varepsilon_{\mathrm{h}}} N_{\mathrm{tot}} \sigma_{21} \frac{W_{\mathrm{p}}}{\gamma_{21}+W_{\mathrm{p}}}
$$

where $N_{\text {tot }}$ is the density of the chromophores and $\sigma_{21}$ is their (orientation-averaged) emission cross section in the bulk host medium. The pumping rate $W_{\mathrm{p}}$ depends on the (local) pumping field $\mathbf{E}_{\mathrm{p}}$ and the chromophore bulk absorption cross section (we assume $\sigma_{30}=\sigma_{21}$ ),

$$
W_{\mathrm{p}}=\frac{c \varepsilon_{0} \sqrt{\varepsilon_{\mathrm{h}}} \sigma_{30}}{2 \hbar \omega_{\mathrm{p}}}\left|\mathbf{E}_{\mathrm{p}}\right|^{2}
$$

where the pumping frequency $\omega_{\mathrm{p}}$ corresponds to a free-space wavelength of $\lambda_{\mathrm{p}}$. We assume that the spaser is tuned close to the $2 \rightarrow 1$ transition of the chromophores, meaning that $\omega_{\mathrm{s}}-$ $\omega_{21} \ll \gamma_{\mathrm{L}}$. The spasing rate $W_{\mathrm{s}}$ can be analogously expressed by replacing $\omega_{\mathrm{p}} \rightarrow \omega_{\mathrm{s}}, \mathbf{E}_{\mathrm{p}} \rightarrow \mathbf{E}_{\mathrm{s}}$ and $\sigma_{30} \rightarrow \sigma_{21}$, where $\mathbf{E}_{\mathrm{s}}$ is the local, surface plasmon (spasing) field. Saturation field $E_{\text {sat }}$ in eq 1 can be expressed as follows from eq 21 in ref 6

$$
E_{\mathrm{sat}}^{2}=\frac{2 \hbar \omega_{\mathrm{s}}\left(\gamma_{21}+W_{\mathrm{p}}\right)}{c \varepsilon_{0} \sqrt{\varepsilon_{\mathrm{h}}} \sigma_{21}}
$$

It can be understood as the strength of the spasing field at which gain saturation effects become significant. For example, at $\left|\mathrm{E}_{\mathrm{s}}\right| \approx \mathrm{E}_{\mathrm{sat}}$ (which corresponds to $W_{\mathrm{s}} \approx W_{\mathrm{p}}+\gamma_{21}$ ), the gain dielectric function (at midline) is halved with respect to the nonsaturated expression. Finally, $\gamma_{21}$ is the Purcell-enhanced spontaneous decay into the resonant mode for the $2 \rightarrow 1$ transition. From quantum-mechanical treatment, ${ }^{7,8}$ we estimate (see Supporting Information, end of the section PurcellEnhanced Spontaneous Emission, and Table 2 for values)

$$
\gamma_{21}=\frac{c \sigma_{21}}{V_{\mathrm{G}} \sqrt{\varepsilon_{\mathrm{h}}}}
$$

where $V_{\mathrm{G}}$ is the effective mode volume in the gain material.

In calculations we used the following parameters, which are typical for organic fluorophores: $\lambda_{21}=520 \mathrm{~nm}, \gamma_{\mathrm{L}}=3.8 \times 10^{14}$ $\mathrm{s}^{-1}, N_{\text {tot }}=8 \times 10^{25} \mathrm{~m}^{-3}, \sigma_{21}=2.5 \times 10^{-20} \mathrm{~m}^{2}$, and $\lambda_{\mathrm{p}}=470$ $\mathrm{nm}$, and the gain host is chosen to be silica with $\varepsilon_{\mathrm{h}}=2.14$. ${ }^{9}$

In detailed analysis of the spaser, ${ }^{10}$ (end of section 2.1 there) a chromophores concentration of $N_{\text {tot }}=2.4 \times 10^{26} \mathrm{~m}^{-3}$ was assumed. Experiments ${ }^{11}$ implied $N_{\text {tot }}=4.7 \times 10^{26} \mathrm{~m}^{-3}$ within a $5 \mathrm{~nm}$ thick doped silica shell. In the current study, we use concentrations, that are a factor of 3-5 less and are easier to achieve experimentally.

While mean-field chromophore interaction is included via a field- and position-dependent dielectric function with gain saturation (1), we do not discuss more complex cooperative effects, ${ }^{12,13}$ assuming that they are smeared out by strong dephasing and random dipole orientations. Indeed, in the recent paper, ${ }^{14}$ Petrosyan and Shahbazyan concluded that with tens of thousands randomly oriented molecules, the ensembleaveraged dipole-dipole coupling vanishes, and the resonant mode is also unaffected by it.

Spasing Threshold. The spasing threshold corresponds to the minimum pumping strength required to start and maintain the generation. For simulations, it is more convenient to express the spasing threshold in terms of the gain level $\varepsilon_{\mathrm{L}}$ and not the pumping rate $W_{\mathrm{p}}$. To find the threshold numerically, we vary the spasing frequency $\omega_{\mathrm{s}}$ and the gain level $\varepsilon_{\mathrm{L}}$ to find the set of parameters $\left(\omega_{\mathrm{thr}}, \varepsilon_{\mathrm{L}, \mathrm{thr}}\right)$, for which the electric field diverges in an unsaturated, linear system (i.e., with $\left(\left|\mathrm{E}_{\mathrm{s}}\right| / E_{\mathrm{sat}}\right)^{2}$ $\rightarrow 0$ in eq 1 , see Arnold et al. $\left.{ }^{15}\right) . \omega_{\text {thr }}$ is called the threshold generation frequency of the spaser, while $\varepsilon_{\mathrm{L} \text {,thr }}$ will be referred to as the gain threshold. After the pair $\left(\omega_{\mathrm{thr}}, \varepsilon_{\mathrm{L}, \mathrm{thr}}\right)$ is found, all simulations are carried out with gain saturation included.

Heat Sources in Spasers. In an operating spaser, the heat released in the metal can be described by the volumetric power of Ohmic losses, $Q$, using the imaginary part of the metal dielectric function $\varepsilon_{M}^{\prime \prime}(\omega)$,

$$
Q(\omega, \mathbf{r})=\frac{1}{2} \omega \varepsilon_{0} \varepsilon_{M}^{\prime \prime}(\omega)|\mathbf{E}(\omega, \mathbf{r})|^{2}
$$

where $\mathbf{E}(\omega, \mathbf{r})$ is the amplitude of the electric field at the location $\mathbf{r}$ inside the metal, either at the spasing or the pumping frequency.

The gain material is heated by nonradiative decay of the chromophores. The corresponding heat source can be described by (see Supporting Information, section Heat Source in the Gain Material)

$$
Q_{\mathrm{G}}=N_{\mathrm{tot}}\left(\hbar \omega_{10}+\hbar \omega_{32}\right) \frac{W_{\mathrm{p}}\left(W_{\mathrm{s}}+\gamma_{21}\right)}{W_{\mathrm{p}}+W_{\mathrm{s}}+\gamma_{21}}
$$

\section{MULTISCALE AND MULTIPHYSICS NUMERICAL MODELING}

Coupling of the Thermal and Electromagnetic Problems. The coupling between the electromagnetic and the thermal problem is achieved via a temperature-dependent 
metal dielectric function (see Supporting Information, section Temperature-Dependent Drude Model, and Reddy et al. ${ }^{16}$ ). To simulate the surface plasmon oscillations in a spaser, we use COMSOL Multiphysics, which is a versatile commercial software package based on the finite element method (FEM). We combine nonlinear Maxwell's equations (solved in the frequency domain) with the heat equation (solved in the time domain) using a so-called frequency-transient study step sequence: first, we solve the electromagnetic problem with gain saturation in frequency domain and use its results (i.e., the heat sources) to make a time step in the thermal problem. Then, we recompute all related quantities (e.g., the temperaturedependent dielectric function) and solve the electromagnetic problem again, using the previous solution as an initial guess. The underlying assumption for the validity of this multiscale and multiphysics computational workflow is that the electromagnetic problem approaches equilibrium much faster than the thermal one. In particular, quantum coherence effects ${ }^{17-20}$ are omitted in our analysis. Polarization adiabatically tracks the populations for times longer than the dephasing time $\gamma_{\mathrm{L}}^{-1}$ (inverse atomic line width). Note that recent experiments, ${ }^{21,22}$ which reveal pronounced coherence effects in plasmonic systems, essentially deal with single emitters, which makes influence of dephasing much less crucial, as opposed to more than 1000 emitters discussed here. Transients in plasmon and population dynamics end on the time scale of Purcellenhanced atomic relaxation time $\gamma_{21}^{-1}$ that is, within dozens of picoseconds at most (Table 2), as vibronic relaxations $\gamma_{32}, \gamma_{10}$ and plasmon decay are even faster. Detailed quantum analysis of spaser in ref 23 shows eqs 33-37 and Figures 4 and 5 in Supporting Information there, and the discussion in eq S8 on pp S4 and S5 of Supporting Information, that generation stabilizes below 1 ps (see also Figure 4a,b in ref 10 ). We study much longer time scales of 10 ps to $1 \mathrm{~ns}$, under constant pumping intensity. Transient ps processes do exist, but the heating during this time is negligible (see Figure 3). Thermal dynamics occurs over larger time scales, where quasi-stationary electrodynamics adiabatically follows slow changes in parameters. Such slowly varying envelope approach fails, if the duration of the transient processes $O(\mathrm{ps})$ becomes comparable with polarization dephasing time $O(\mathrm{fs})$. Fortunately, this is almost never the case.

The electrodynamic simulations of the pumping field are carried out at pumping frequency $\omega_{\mathrm{p}}$, and they must be decoupled from the spasing simulations (which are performed at spasing frequency $\omega_{\mathrm{s}}$ ). Equation 1 describes the emission properties of chromophores near the spasing frequency, but there is also a Lorentzian with opposite sign around $\omega_{30}=\omega_{\mathrm{p}}$ (i.e., the $3 \rightarrow 0$ transition of the dyes), which corresponds to the absorption cross section. Then, the gain dielectric function for the pumping simulation is ${ }^{6}$

$$
\varepsilon_{\mathrm{G}}\left(\omega_{\mathrm{p}}\right)=\varepsilon_{\mathrm{h}}+i \frac{c \sqrt{\varepsilon_{\mathrm{h}}} N_{\mathrm{tot}} \sigma_{30} / \omega_{\mathrm{p}}}{1+\left(\left|\mathbf{E}_{\mathrm{p}}\right| / E_{\mathrm{p}, \mathrm{sat}}\right)^{2}}
$$

where $E_{\mathrm{p}, \text { sat }}$ is the local pumping saturation field,

$$
E_{\mathrm{p}, \mathrm{sat}}^{2}=\frac{2 \hbar \omega_{\mathrm{p}}\left(\gamma_{21}+W_{\mathrm{s}}\right)}{c \varepsilon_{0} \sqrt{\varepsilon_{\mathrm{h}}} \sigma_{30}}
$$

The heat released in the gain material is already addressed by eq 7 (which is implemented in the spasing simulation); thus, we only have to consider the heat released in the metal.
For computational efficiency, the problem can then be further decoupled: the temperature only needs picoseconds to spread over the metal due to its high thermal diffusivity, but the thermal problem needs several nanoseconds to reach the stationary solution. The high thermal conductivity of the metal further means that the temperature gradient is very small there. Thus, we can assume a homogeneous metal temperature and run many purely electrodynamic pumping simulations for various metal dielectric functions $\varepsilon_{M}(T)$, where $T$ varies within $300-1200 \mathrm{~K}$. From these results, we can then build a temperature-dependent pumping heat source $Q_{p}(T)$ (see eq 6 ). For example, the metal dielectric function at temperature $T$ is $\varepsilon_{M}(T)$, and the average power of the pumping heat source per unit volume is

$$
Q_{\mathrm{p}}(T)=\frac{\omega \varepsilon_{0}}{2 V_{\mathrm{M}}} \int_{\mathrm{M}} \mathrm{d} V \varepsilon_{\mathrm{M}}^{\prime \prime}(T)\left|\mathbf{E}_{\mathrm{p}}\right|^{2}
$$

where $V_{\mathrm{M}}$ is the metal volume and $\mathrm{E}_{\mathrm{p}}$ is the local pumping field induced by an incident (plane wave) intensity $I_{\text {in }}$ (which is the same in all simulations). If done thoroughly for an array of temperatures (steps on the order of $1 \mathrm{~K}$ ), this method improves convergence and is in good agreement with the results from a continuously coupled electromagnetic/thermal numerical model.

Kapitza Resistance. In the thermal simulations, we employ the Kapitza resistance ${ }^{24,25}$ in a form of interfacial thermal boundary condition. Due to the Kapitza resistance, which is essential for nanostructures, the temperature is discontinuous at the interface between two materials. The step in temperature is proportional to the heat flux $J$ across the boundary $S$,

$$
\left.J\right|_{S}=G\left(T_{1}-T_{2}\right)
$$

where $T_{1}$ and $T_{2}$ are the corresponding surface temperatures on either side of the interface. $G$ is called interfacial thermal conductance and depends on the materials and type of interface (e.g., metal/water, metal/metal, ...). The stationary temperature distribution around a continuously heated, spherical nanoparticle provides an estimation of the importance, and good motivation for the inclusion of the Kapitza resistance. The relative change in temperature at the boundary in this case is (see Supporting Information, section The Stationary Solution for a Continuously Heated, Spherical Nanoparticle with Kapitza Resistance)

$$
\frac{T_{1}-T_{2}}{T_{2}}=\frac{k_{\mathrm{a}}}{G a}
$$

where $a$ is the nanoparticle radius and $k_{\mathrm{a}}$ is the thermal conductivity of the ambient. Thus, the smaller the nanoparticle, the larger is the influence of the Kapitza resistance.

Geometry, Materials, and Pumping. To explore the operational thermodynamics of example spaser designs, we choose them from a family of coaxial oblate or prolate spheroidal core-shell configurations with a variable core aspect ratio $\kappa>1$. The pumping field can be parallel or perpendicular to the axis of revolution. The shell spheroid is defined with a shell thickness $h$ : for a core spheroid with a major semiaxis $a$ and a minor semiaxis $a / \kappa$, the major and minor semiaxes of the shell will be $a+h$ and $a / \kappa+h$, respectively. Examples for $a=h=30 \mathrm{~nm}, \kappa=6.25$ and $a=h=$ $23 \mathrm{~nm}, \kappa=1.94$ are shown in Figure 2, panels a, b and c, d, respectively. 
(a)
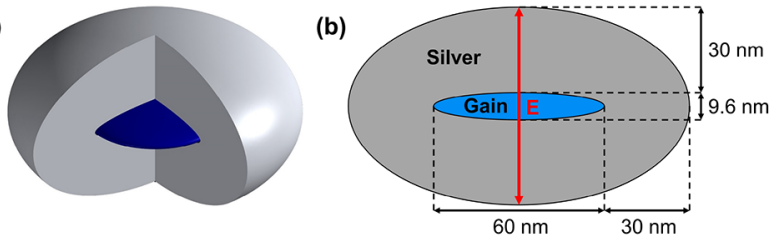

(c)

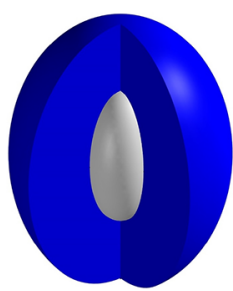

(d)

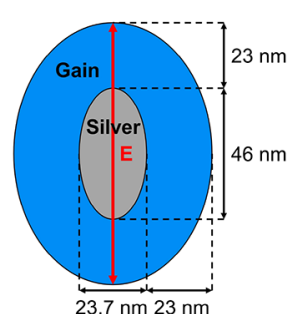

Figure 2. Geometries of the investigated spasers. Both spasers are numerically tuned to a resonant wavelength of $520 \mathrm{~nm}$ via variation of their core aspect ratios. The polarization of the pumping field (denoted E) is parallel to the axis of revolution. (a) and (b) The oblate gain-core/metal-shell configuration has a core aspect ratio $\kappa=$ 6.25. (c, d) Prolate metal-core/gain-shell configuration has a core aspect ratio $\kappa=1.94$. For both configurations the ambient material is water.

To examine the thermal dynamics of the metal-core/gainshell and gain-core/metal-shell configuration, it is important to clarify some basic specifications for both structures. We choose silver for metal components (dielectric function by Johnson and Christy $\left.^{35}\right)$ and silica $\left(\varepsilon_{\mathrm{h}}=2.14^{9}\right)$ as gain host material. Both spasers are immersed in water $\left(\varepsilon_{\mathrm{a}}=1.78^{36}\right)$ to imitate realistic experimental conditions. Both spasers have similar dimensions, low gain thresholds $\left(\varepsilon_{\mathrm{L}, \mathrm{thr}}<0.15\right)$, and the same generation frequency, which lies in the visible range. Table 1

Table 1. Room Temperature Values of Thermal Material Properties (Thermal Conductivity $k$, Specific Heat Capacity $c$, Density $\rho)$ for Silver $(\mathrm{Ag})$, Silica $\left(\mathrm{SiO}_{2}\right)$, and Water $\left(\mathrm{H}_{2} \mathrm{O}\right)$

\begin{tabular}{clccc} 
& & $\mathrm{Ag}^{26-28}$ & $\mathrm{SiO}_{2}{ }^{29-31}$ & $\mathrm{H}_{2} \mathrm{O}^{32-34}$ \\
$k$ & {$[\mathrm{~W} /(\mathrm{m} \cdot \mathrm{K})]$} & 426.4 & 2.40 & 0.61 \\
$c$ & {$[\mathrm{~J} /(\mathrm{kg} \cdot \mathrm{K})]$} & 236.7 & 748 & 4181.5 \\
$\rho$ & {$\left[\mathrm{kg} / \mathrm{m}^{3}\right]$} & 10470 & 2196 & 998 \\
\hline
\end{tabular}

shows the room temperature values for the thermal material properties. With the exception of the Kapitza conductance, all material properties are assumed to be temperature-dependent. The values for the Kapitza conductance vary widely in the literature, ${ }^{37-43}$ so we choose a temperature-independent Kapitza conductance $G=10^{8} \mathrm{~W} /\left(\mathrm{m}^{2} \cdot \mathrm{K}\right)$ for all interfaces, which is close to $6 \times 10^{7} \mathrm{~W} /\left(\mathrm{m}^{2} \cdot \mathrm{K}\right)$ found in our recent thermoreflectance experiments. ${ }^{44}$

From quasistatic results (see Supporting Information, section Quasistatic Spasing Condition for Confocal Spheroidal Core-Shell Structures), we find that three spaser configurations have low gain thresholds $\left(\varepsilon_{\mathrm{L}, \mathrm{thr}}<0.15\right)$ and generation frequencies in the visible range. For the final comparison, we choose to simulate only two essentially different geometries with respect to shape and order of materials: the oblate gaincore/metal-shell structure with $\mathbf{E} \| \mathbf{z}$ and the prolate metalcore/gain-shell structure with $\mathbf{E} \| \mathbf{z}$, where $\mathbf{z}$ represents the axis of revolution. For the excitation field used, both structures show rotational symmetry, which is convenient for simulations. The omitted third oblate spaser with the nonsymmetric

excitation along the large axis also has marginally higher threshold than these two. The spasers are tuned to a generation wavelength of $520 \mathrm{~nm}$ via variation of their core aspect ratio. At $520 \mathrm{~nm}$, the gain threshold has a local minimum and the aspect ratios are realistic (i.e., the structures are not unrealistically thin). We set the pumping frequency $\omega_{\mathrm{p}}$ to $470 \mathrm{~nm}$, which corresponds to a Stokes difference typical for organic dyes in this spectral range. In ref 10 (middle of the section 1.1 there), a spaser with a (spherical) gain core of radius $12 \mathrm{~nm}$ and metal shell thickness below $1 \mathrm{~nm}$ are discussed. In the case of manufacturing issues, a simple suggestion to scale the device up by a factor of 2 or 3 could be offered. Our studies reveal that such an easy fix will not work for several reasons: retardation, out-radiation, and thermal effects. To provide the guidelines for the experiments, we kept the wavelength, metal and gain thicknesses and the chromophore concentration as realistic as possible. This unavoidably leads to larger sizes discussed here, which can be also easier to manufacture. The resulting spaser geometries are shown in Figure 2. Such numbers and geometries are feasible for modern methods of dye-matrix manufacturing (for instance, using stimulated emission depletion (STED) lithography ${ }^{45}$ ). The values of the numeric spasing thresholds are listed in Table 2 among other operational parameters.

Table 2. Operational Parameters for the Gain-Core/MetalShell (Gain/Metal) and the Metal-Core/Gain-Shell (Metal/ Gain) Spaser in Comparison ${ }^{a}$

$\begin{array}{llll} & & \text { gain/metal } & \text { metal/gain } \\ \varepsilon_{\mathrm{L}} & {[1]} & 0.193 & 0.197 \\ \varepsilon_{\mathrm{L}, \mathrm{thr}} & {[1]} & 0.113 & 0.116 \\ \lambda_{\text {thr }} & {[\mathrm{nm}]} & 519.96 & 519.78 \\ W_{\mathrm{p}}^{\dagger} & {[1 / \mathrm{s}]} & 1.10 \times 10^{12} & 1.02 \times 10^{11} \\ W_{\mathrm{s}}^{\dagger} & {[1 / \mathrm{s}]} & 1.08 \times 10^{12} & 2.29 \times 10^{10} \\ \gamma_{21} & {[1 / \mathrm{s}]} & 2.83 \times 10^{11} & 2.32 \times 10^{10} \\ I_{\text {in }} & {\left[\mathrm{W} / \mathrm{cm}^{2}\right]} & 2.16 \times 10^{7} & 5.39 \times 10^{7}\end{array}$

${ }^{a}$ Quantities marked with ${ }^{\dagger}$ depend on position and time: they are averaged over the gain material and evaluated at $t=0$ (which corresponds to $T=300 \mathrm{~K}$ ).

The pumping strength of a spaser is a key parameter for its operation. It can be expressed in terms of several quantities (which are all related to each other): (i) the pumping rate $W_{\mathrm{p}}$, (ii) the gain level $\varepsilon_{\mathrm{L}}$, or (iii) the incident pumping intensity $I_{\text {in }}$ of a focused pumping laser, which induces the field $\mathbf{E}_{\mathrm{p}}$ in eq 3 . We are interested in the case of post-threshold pumping, thus we simulate our spasers at the gain level $\varepsilon_{\mathrm{L}}=1.7 \varepsilon_{\mathrm{L}, \mathrm{thr}}$. The corresponding parameters $\left(W_{\mathrm{p}}, I_{\mathrm{in}}, \ldots\right)$ can be found in Table 2 .

Thermal Limits. There are several factors that determine the thermal limitations of a spaser. Generally, a deformation of the geometry should be avoided, which means that the operating temperature should stay below the melting point of the gain host and the metal, respectively. However, Inasawa et al. $^{46}$ show that laser-induced surface reshaping of gold nanoparticles occurs approximately 120 degrees below the melting point. The melting point of silver is at $1235 \mathrm{~K},{ }^{31}$ which yields an estimated reshaping temperature of around $1115 \mathrm{~K}$. To be on the safe side, we set the maximally allowed metal temperature to $1000 \mathrm{~K}$.

Also critical is the thermal decomposition of the chromophores: if the dye molecules are thermally damaged, the changes of the absorption and emission spectra are 
irreversible and the principal functionality of the spaser is threatened. The thermal decomposition temperatures of certain dyes can be quite low, for example, rhodamine B decomposes at around $520-570 \mathrm{~K}^{47,48}$ To safely avoid thermal damage of the chromophores, the temperature of the gain material should not exceed $500 \mathrm{~K}$, which makes this a dominant limitation for the combination of materials used here. Since silica has a melting point of $1986 \mathrm{~K}^{31}$ this condition also prevents melting effects in the gain host.

\section{RESULTS}

Figure 3 shows the results for the average and maximum temperatures over time in the respective spaser components.
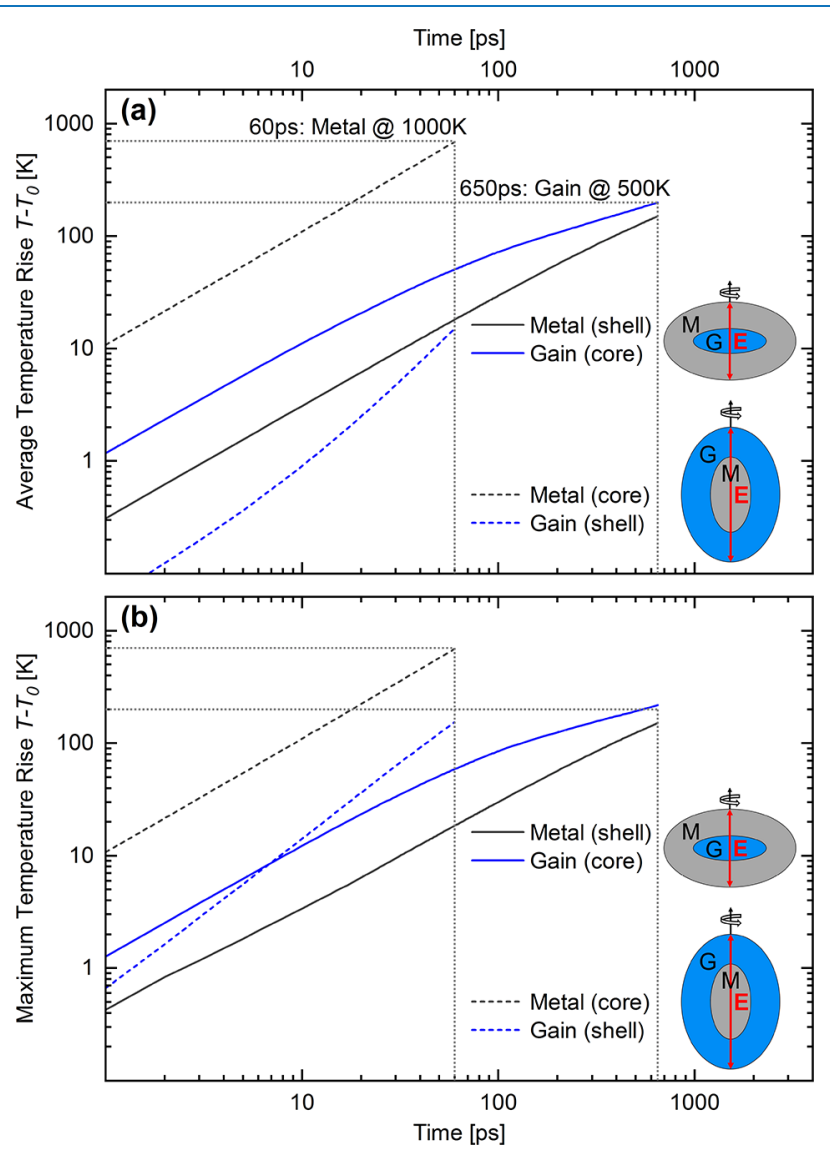

Figure 3. (a) Average and (b) maximum temperature rise $T-T_{0}$ in the respective spaser components (metal and gain). Solid curves are for the oblate gain-core/metal-shell, dashed curves for the prolate metal-core/gain-shell spaser. The maximum operation time is 650 and $60 \mathrm{ps}$, respectively (see section Thermal Limits for the thermal limits). Due to the high thermal conductivity of silver, there is practically no difference between average and maximum temperature in the metal.

The oblate gain-core/metal-shell spaser can operate up to 650 ps under the specified pumping conditions before the gain host reaches the critical temperature of $500 \mathrm{~K}$. For the prolate metal-core/gain-shell spaser, the metal component is the limiting factor: after approximately $60 \mathrm{ps}$, the metal core reaches a temperature of $1000 \mathrm{~K}$ and spaser operation needs to be terminated to avoid melting or surface reshaping effects. Thus, the maximum pulsed operation times are 650 and $60 \mathrm{ps,}$ respectively. For information regarding the cooling of the structures on a nanosecond time scale, see Supporting Information, section Cooling Time.
Figure 4 shows the distribution of the spasing field $\left|E_{s}\right|$ at the end of the pumping pulse $(650$ or $60 \mathrm{ps})$ in both

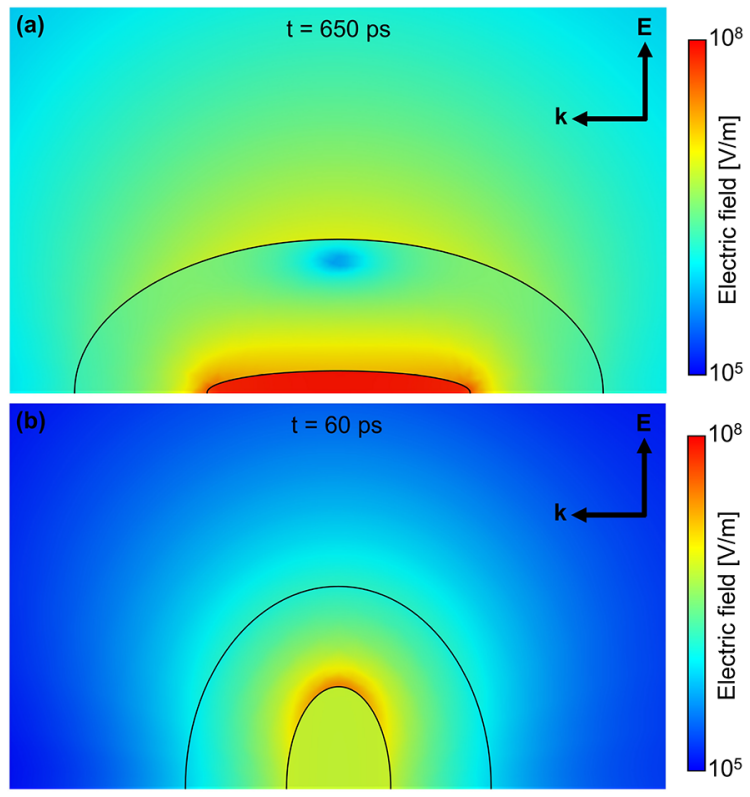

Figure 4. Distribution of the spasing field $\left|E_{s}\right|$ at the end of the pumping pulse in (a) the oblate gain-core/metal-shell and (b) the prolate metal-core/gain-shell spaser. Wavevector $\mathbf{k}$ refers to the pumping field, while $\mathbf{E}$ indicates the prevalent polarization of both pumping and spasing fields.

configurations. The spasing mode is dipolar in both cases. For the gain-core/metal-shell geometry, the field is approximately an order of magnitude stronger in the core, which leads to more effective chromophore utilization. The field in the oblate gain core is quite homogeneous, and with the increasing size, such a design transforms into a metal-clad nanolaser, 4,49 operating on the first TM mode (dipolar Mie void plasmon in the case of a spherical core-shell). Thus, our analysis bridges the gap between spasers and nanolasers. The small dip in the electric field within the shell of the gain-core/metal-shell oblate spaser is most likely a needle effect: the large curvature along the equator of the "pancake" (left and right sides of the structure in Figure 4a) leads to the disproportionately large fields in this region. The dip is very shallow: the field falls approximately $15 \%$.

Figure 5 shows the temperature distribution at the end of the pumping pulse ( 650 or $60 \mathrm{ps}$ ). Due to the high thermal conductivity of silver, the metal temperature is spatially almost constant in both configurations. In the metal-core/gain-shell spaser, the gain-shell acts as a thermally insulating layer: after $60 \mathrm{ps}$, the heat wave has penetrated only the first $13 \mathrm{~nm}$ of the approximately $23 \mathrm{~nm}$ thick gain-shell. In order to diffuse through the shell and reach the ambient, the heat wave would need approximately 175 ps, much longer than the maximally allowed pulse length. For the gain-core geometry, metal is in direct contact with water, which represents the ultimate heat sink. Since most of the heat is generated inside the metal, the operation temperature in this structure is much lower than in its counterpart. Because of the Kapitza resistance, the temperature jumps at the metal-gain interface: in Figure 5, the discontinuity is $40 \mathrm{~K}$ for the gain-core/metal-shell and 540 $\mathrm{K}$ for the metal-core/gain-shell spaser. The average curvature 

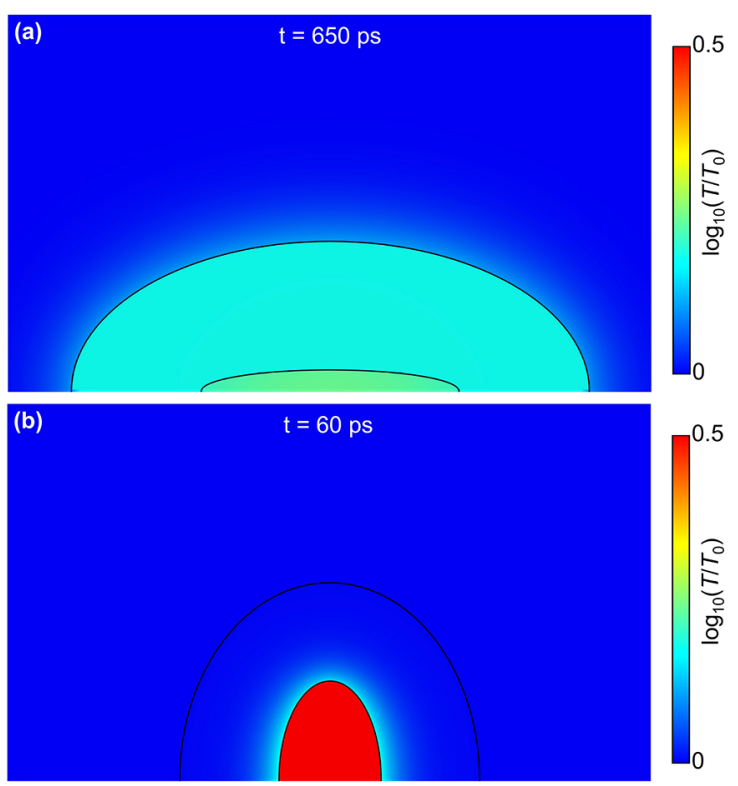

Figure 5. Temperature distribution at the end of the pumping pulse in (a) the oblate gain-core/metal-shell and (b) the prolate metalcore/gain-shell spaser. For the latter, the shell acts as a thermally insulating layer. Note that the scale is logarithmic from $T_{0}=300 \mathrm{~K}$ to $T \approx 950 \mathrm{~K}$.

of the prolate spaser is larger and its temperature is higher, hence, the temperature discontinuity is more pronounced.

We apply the thermal restrictions of section Thermal Limits to the average rather than the maximum temperature. For the metal component, the choice is irrelevant: the temperature difference over the volume is of the order of $1 \mathrm{~K}$, and the average and maximum temperatures largely coincide (black curves in Figure 3). In the gain material, however, intense temperatures can occur at the gain/metal interface (especially if the gain shell acts as an insulating layer). The affected area is very small though (only the first few $\mathrm{nm}$, see Figure $5 \mathrm{~b}$ ), so slight melting effects or chromophore decomposition are almost negligible. In fact, thermal destruction of chromophores in close proximity to the metal might even be beneficial: Kewes et al. ${ }^{23}$ argue that a nonactive layer of a few nanometers around a metal core reduces quenching (i.e., the excitation of undesired, higher-order surface plasmon modes). In real systems, the heat generated by the Ohmic decay of higherorder surface plasmons may be significant, thus, a nonactive "spacing" layer can prove thermally advantageous.

The contributions of different heat souces to the total heating power in the spasers are shown in Figure 6a. For the gain-core/metal-shell spaser (solid curves), the heat induced by the spasing (at $520 \mathrm{~nm}$ ) and pumping (at $470 \mathrm{~nm}$ ) fields is comparable, while for the metal-core/gain-shell spaser (dashed curves), the pumping field heat source is the dominating contribution. In both cases, the heat generated by vibronic relaxation of the chromophore excitation is negligible compared to the total heating power. Though metal absorption at pumping wavelength is the largest heat source, the results show that pulsed operation of both devices is feasible.

The opposite temporal behavior of the spasing and pumping heating powers in Figure $6 \mathrm{a}$ is due to an increase in temperature with time. To understand both trends, we have to look at the temperature dependence of the Ohmic loss, eq 6: $Q(T) \propto \varepsilon_{M}^{\prime \prime}(T)|\mathbf{E}(T)|^{2}$. The imaginary part of the metal
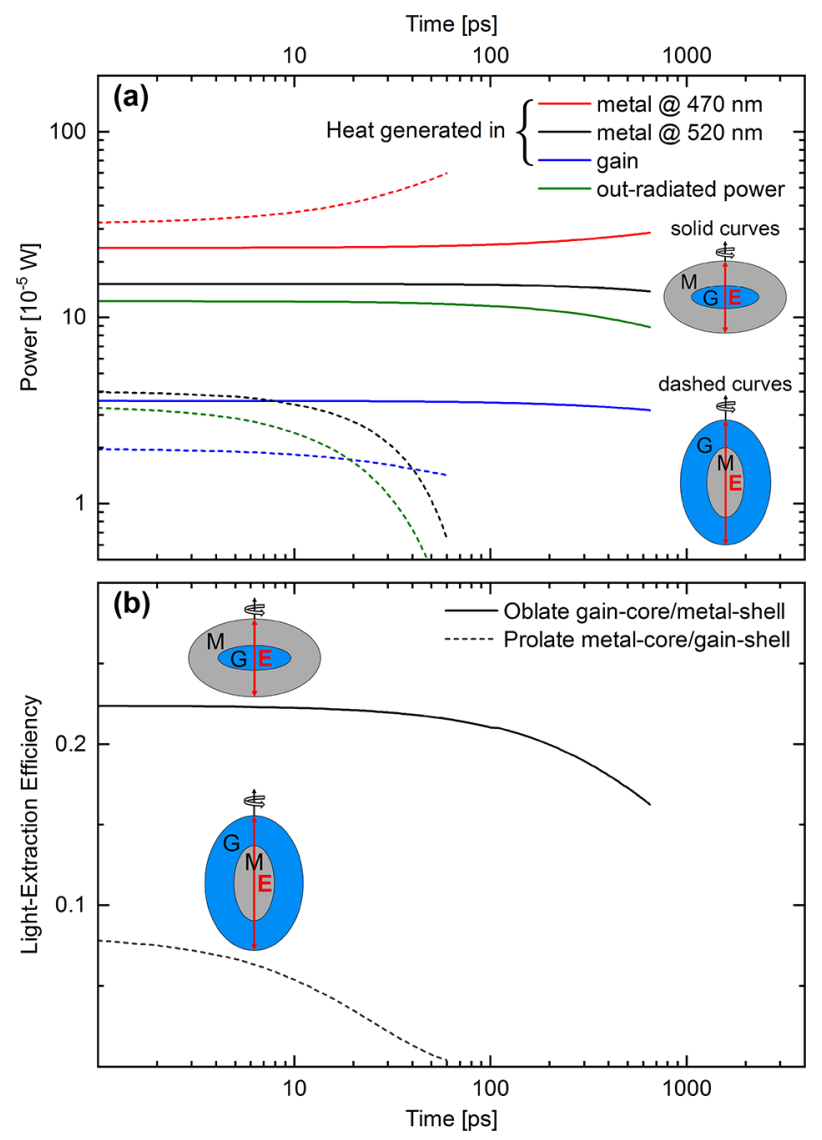

Figure 6. (a) Heating power (integrated heat sources) in the respective spaser components and the out-radiated power (integrated far-field Poynting vector of the spasing field) for both spasers: oblate gain-core/metal-shell (solid curves) and prolate metal-core/gain-shell (dashed curves). The maximum operation times are 60 and 650 ps. The heat generated at the spasing/pumping frequency in the metal is induced by the spasing/pumping field $\left(\mathbf{E}_{\mathrm{s}}, \mathbf{E}_{\mathrm{p}}\right)$, respectively. (b) Internal light-extraction efficiency of both spasers, calculated as described in the text.

dieletric function increases linearly with temperature, $\varepsilon_{\mathrm{M}}^{\prime \prime} \propto C_{1}$ $+C_{2} T$, while $\varepsilon_{M}^{\prime}$ remains effectively constant (see Supporting Information, section Temperature-Dependent Drude Model). The pumping field, which is off-resonant, is largely dominated by the absolute value of the dielectric function, which in turn remains approximately constant since $\left|\varepsilon_{M}^{\prime}\right| \gg\left|\varepsilon_{M}^{\prime \prime}\right| \Rightarrow\left|\varepsilon_{M}\right| \approx$ $\left|\varepsilon_{M}^{\prime}\right| \approx \operatorname{const}(T)$. Thus, the pumping field $\mathbf{E}_{\mathrm{p}}$ depends only weakly on temperature and the pumping heat source $Q_{p}$ increases linearly with temperature, since

$$
Q_{\mathrm{p}}(T) \propto \varepsilon_{\mathrm{M}}^{\prime \prime}(T)
$$

The temperature dependence of the spasing field in the metal can be qualitatively understood using the quasistatic estimation for the gain-core/metal-shell spaser at the threshold generation frequency $\omega_{\text {thr }}$ (see eq S32 in Supporting Information, section Quasistatic Estimation for the Temperature Dependence of the Spasing Field at the Threshold Generation Frequency, and the discussion there),

$$
\left|\mathbf{E}_{\mathrm{s}}\left(\omega_{\mathrm{thr}}, T\right)\right|^{2} \propto E_{\mathrm{sat}}^{2}\left(\frac{\varepsilon_{\mathrm{L}}\left|\varepsilon_{\mathrm{M}}^{\prime}\right|}{\varepsilon_{\mathrm{h}} \varepsilon_{\mathrm{M}}^{\prime \prime}(T)}-1\right)
$$

The saturation field $E_{\text {sat }}$ and the gain level $\varepsilon_{\mathrm{L}}$ are related to the pumping field $\mathbf{E}_{\mathrm{p}}$, see eqs $2-4$, and depend only weakly on 
temperature. Thus, the spasing heat source $Q_{s}$ decreases almost linearly with temperature,

$$
Q_{\mathrm{s}}(T) \propto \frac{\varepsilon_{\mathrm{L}} \mid \varepsilon^{\prime}{ }_{\mathrm{M}} \mathrm{l}}{\varepsilon_{\mathrm{h}}}-\varepsilon^{\prime \prime}{ }_{\mathrm{M}}(T)
$$

Additionally, in Figure 6a, the farfield out-radiated power (green curves) is shown. For both geometries, it is comparable with the absorption of the spasing field by the metal (black curves). This contrasts with the often used quasistatic assumption, that the dominant losses of the generating spaser are purely absorptive. ${ }^{10}$ Such an approximation indeed holds for a spaser of few $\mathrm{nm}$ in size, but for a realistic geometry with sizes around $100 \mathrm{~nm}$ studied here, the retardation effects and radiative losses cannot be neglected. Here, the radiative losses equal about $80 \%$ of the absorption losses, which is in qualitative agreement with the numerical gain thresholds for our spasers, which are about $60-70 \%$ higher than the quasistatic predictions (see Supporting Information, section Quasistatic Spasing Condition for Confocal Spheroidal CoreShell Structures). The relation between the absorption and outradiation holds both near and above threshold, when gain saturation sets in, because both are proportional to the square of the spasing field. In an idealized laser with negligible absorptive losses, post-threshold pumping is fully converted into useful output (not counting the Stokes shift). In a spaser, the field in the metal represents an immanent part of the resonant mode, and the out-radiation and Ohmic losses maintain a constant ratio even above threshold.

Importantly, the results in Figure 6a allow for the estimation of the farfield emission efficiency for both spasers. Within the employed approximations, the overall incoming absorbed power is converted into the losses in the metal at the pumping and spasing wavelengths, the losses in the gain material, and the out-radiated power, that is, equals the sum of all four curves in Figure 6a (quenching and scattering at the pumping wavelength are neglected here). Dividing the radiated power (green curves) by this sum, we arrive at an internal farfield light-extraction efficiency, which is shown in Figure 6b. At early times, when the temperature is low, it reaches $22.4 \%$ and $8.1 \%$ for the oblate and prolate geometries, respectively. While for the prolate geometry, the efficiency degrades to half of the initial value already after only about $16 \mathrm{ps}$, for the oblate case with the gain core, it remains almost constant, and is still equal to $16.3 \%$ at the thermally defined time limit of of $650 \mathrm{ps}$. This is not overly surprising, as with increasing size this structure becomes similar to a metal-clad nanolaser. ${ }^{4,49}$

The decrease in the radiated power is concomitant with the overall decrease of the spasing field with temperature, and therefore time (see eq 14). For the dipolar modes used here and the numbers considered, the oblate geometry with gain core turns out to be a better farfield emitter of light than the prolate one, both in absolute numbers and in terms of extraction efficiency. This is related to the overall better performance and larger spasing fields there (see Figure 4) and, possibly, to the nature of the metal shell plasmon in this case (low-energy, symmetric, bonding), where surface charges on the outer and inner surfaces of the shell oscillate in phase. ${ }^{50}$ Larger out-radiated power (losses) is a desired outcome if a spaser is used as a nanoscopic light source. For the applications where it is employed as a local coherent field amplifier, for instance, for surface-enhanced Raman scattering (SERS), larger local fields are of higher relevance, despite associated absorption in the metal. Here, prolate geometry, or higher order modes with smaller radiative losses may be preferable.

We also simulated similar spasers with polystyrene as gain host material, which was recently used as a matrix for organic dyes in microresonators. ${ }^{51-53}$ The summary of these results can be found in the Supporting Information, section Polystyrene as Gain Host Material.

\section{CONCLUSIONS}

We have shown that pulsed operation of spasers with durations longer than $10 \mathrm{ps}$ is principally possible with regard to thermal stability. In particular, the considered core-shell configurations with resonances near $520 \mathrm{~nm}$ can be operated with pulse lengths up to $60 \mathrm{ps}$ (prolate metal-core/gain-shell) and $650 \mathrm{ps}$ (oblate gain-core/metal-shell) in an aqueous ambient. The latter geometry, which with increasing size becomes similar to a metal-clad nanolaser, provides better utilization of gain material, and the metal/water interface proves to be very efficient in transporting heat to the water ambient, which acts as a heat sink. Further, the optically pumped oblate gain core/ metal shell spaser, operated as a nanoscopic coherently radiating light source, reaches an internal light-extraction efficiency of $22.4 \%$, which stays almost constant up until the thermal time limit of 650 ps.

\section{ASSOCIATED CONTENT}

\section{Supporting Information}

The Supporting Information is available free of charge on the ACS Publications website at DOI: 10.1021/acsphotonics.8b00705.

Detailed discussions and derivations on several theoretical and numerical aspects, as mentioned throughout the text (PDF).

\section{AUTHOR INFORMATION}

\section{Corresponding Author}

*E-mail: nikita.arnold@jku.at.

ORCID

Nikita Arnold: 0000-0003-3673-1772

Thomas A. Klar: 0000-0002-1339-5844

\section{Present Address}

${ }^{\S}$ Institute of Experimental Physics/Soft Matter Physics, Johannes Kepler University, Altenberger Straße 69, 4040, Linz, Austria.

\section{Funding}

Funding was provided by the European Research Council (ERC Starting Grant 257158 “Active NP”). A.V.K. acknowledges the support by the DARPA/DSO Extreme Optics and Imaging (EXTREME) Program, Award HR00111720032, and a partial support by the Office of Naval Research Multidisciplinary University Research Initiative (ONR MURI) Grant "Novel Nonlinear Optical Processes in Active, Random and Nanostructured Systems" MURI N00014-13-0649. N.A. thanks the Linz Institute of Technology (LIT), project ADAPT, and the Austrian Science Fund (FWF), Project P 29603, for financial support. G.V.K. acknowledges funding of the Austrian Marshall Plan Foundation and the Internationalisierungsprogramm für Studierende of Upper Austria.

\section{Notes}

The authors declare no competing financial interest. 


\section{ACKNOWLEDGMENTS}

The authors thank Calin Hrelescu (Trinity College, Dublin) for useful discussions and Johann Messner (JKU, Linz) for support with supercomputing infrastructure.

\section{REFERENCES}

(1) Bergman, D. J.; Stockman, M. I. Surface Plasmon Amplification by Stimulated Emission of Radiation: Quantum Generation of Coherent Surface Plasmons in Nanosystems. Phys. Rev. Lett. 2003, 90, 027402 .

(2) Ndukaife, J. C.; Kildishev, A. V.; Nnanna, A. G. A.; Shalaev, V. M.; Wereley, S. T.; Boltasseva, A. Long-range and rapid transport of individual nano-objects by a hybrid electrothermoplasmonic nanotweezer. Nat. Nanotechnol. 2016, 11, 53.

(3) Pissuwan, D.; Valenzuela, S. M.; Cortie, M. B. Therapeutic possibilities of plasmonically heated gold nanoparticles. Trends Biotechnol. 2006, 24, 62-67.

(4) Smalley, J. S. T.; Gu, Q.; Fainman, Y. Temperature Dependence of the Spontaneous Emission Factor in Subwavelength Semiconductor Lasers. IEEE J. Quantum Electron. 2014, 50, 175-185.

(5) Fedorov, I. A.; Parfenyev, V. M.; Vergeles, S. S.; Tartakovsky, G. T.; Sarychev, A. K. Allowable Number of Plasmons in Nanoparticle. JETP Lett. 2014, 100, 530-534.

(6) Arnold, N.; Piglmayer, K.; Kildishev, A. V.; Klar, T. A. Spasers with retardation and gain saturation: electrodynamic description of fields and optical cross-sections. Opt. Mater. Express 2015, 5, 25462577.

(7) Yariv, A. Quantum Electronics, 3rd ed.; John Wiley \& Sons, New York, 1989.

(8) Khurgin, J. B.; Sun, G. Comparative analysis of spasers, verticalcavity surface-emitting lasers and surface-plasmon-emitting diodes. Nat. Photonics 2014, 8, 468-473.

(9) Malitson, I. H. Interspecimen Comparison of the Refractive Index of Fused Silica. J. Opt. Soc. Am. 1965, 55, 1205-1209.

(10) Stockman, M. I. The spaser as a nanoscale quantum generator and ultrafast amplifier. J. Opt. 2010, 12, 024004.

(11) Noginov, M. A.; Zhu, G.; Belgrave, A. M.; Bakker, R.; Shalaev, V. M.; Narimanov, E. E.; Stout, S.; Herz, E.; Suteewong, T.; Wiesner, U. Demonstration of a spaser-based nanolaser. Nature 2009, 460, $1110-1112$.

(12) Bordo, V. G. Cooperative effects in spherical spasers: Ab initio analytical model. Phys. Rev. B: Condens. Matter Mater. Phys. 2017, 95, 235412.

(13) Shesterikov, A. V.; Gubin, M. Y.; Karpov, S. N.; Prokhorov, A. V. On the Effect of Dipole-Dipole Interactions on the Quantum Statistics of Surface Plasmons in Multiparticle Spaser Systems. JETP Lett. 2018, 107, 435-439.

(14) Petrosyan, L. S.; Shahbazyan, T. V. Spaser quenching by offresonant plasmon modes. Phys. Rev. B: Condens. Matter Mater. Phys. 2017, 96, 075423.

(15) Arnold, N.; Hrelescu, C.; Klar, T. A. Minimal spaser threshold within electrodynamic framework: Shape, size and modes. Ann. Phys. 2016, 528, 295-306.

(16) Reddy, H.; Guler, E.; Chaudhuri, K.; Dutta, A.; Kildishev, A. V.; Shalaev, V. M.; Boltasseva, A. Temperature-Dependent Optical Properties of Single Crystalline and Polycrystalline Silver Thin Films. ACS Photonics 2017, 4, 1083-1091.

(17) Kumarapperuma, L.; Premaratne, M.; Jha, P. K.; Stockman, M. I.; Agrawal, G. P. Complete characterization of the spasing (L-L) curve of a three-level quantum coherence enhanced spaser for design optimization. Appl. Phys. Lett. 2018, 112, 201108.

(18) Jha, P. K.; Wang, Y.; Ren, X. X.; Zhang, X. Quantumcoherence-enhanced transient surface plasmon lasing. J. Opt. 2017, 19, 054002 .

(19) Gegg, M.; Richter, M. Efficient and exact numerical approach for many multi-level systems in open system CQED. New J. Phys. 2016, 18, 043037.
(20) Voronine, D. V.; Huo, W. G.; Scully, M. Ultrafast dynamics of surface plasmon nanolasers with quantum coherence and external plasmonic feedback. J. Opt. 2014, 16, 114013.

(21) Gross, H.; Hamm, J. M.; Tufarelli, T.; Hess, O.; Hecht, B. Near-field strong coupling of single quantum dots. Science Advances 2018, 4, eaar4906.

(22) Kongsuwan, N.; Demetriadou, A.; Chikkaraddy, R.; Benz, F.; Turek, V. A.; Keyser, U. F.; Baumberg, J. J.; Hess, O. Suppressed Quenching and Strong-Coupling of Purcell-Enhanced Single-Molecule Emission in Plasmonic Nanocavities. ACS Photonics 2018, 5, $186-191$.

(23) Kewes, G.; Herrmann, K.; Rodríguez-Oliveros, R.; Kuhlicke, A.; Benson, O.; Busch, K. Limitations of Particle-Based Spasers. Phys. Rev. Lett. 2017, 118, 237402.

(24) Kapitza, P. L. Heat Transfer and Superfluidity of Helium II. Phys. Rev. 1941, 60, 354-355.

(25) Swartz, E. T.; Pohl, R. O. Thermal boundary resistance. Rev. Mod. Phys. 1989, 61, 605-668.

(26) Suh, I. K.; Ohta, H.; Waseda, Y. High-temperature thermal expansion of six metallic elements measured by dilatation method and X-ray diffraction. J. Mater. Sci. 1988, 23, 757-760.

(27) Kelley, K. K. High-Temperature Heat-Content, Heat-Capacity, and Entropy Data for the Elements and Inorganic Compounds, 1st ed.; U.S. Government Printing Office: WA, 1960.

(28) Powell, R. W.; Ho, C. Y.; Liley, P. E. Thermal Conductivity of Selected Materials. Technical Report, 1966.

(29) Lord, R. C.; Morrow, J. C. Calculation of the Heat Capacity of $\alpha$ Quartz and Vitreous Silica from Spectroscopic Data. J. Chem. Phys. 1957, 26, 230-232.

(30) Jund, P.; Jullien, R. Molecular-dynamics calculation of the thermal conductivity of vitreous silica. Phys. Rev. B: Condens. Matter Mater. Phys. 1999, 59, 13707-13711.

(31) Haynes, W. M.; Lide, D. R.; Bruno, T. J. CRC Handbook of Chemistry and Physics, 97th ed.; CRC Press: Boca Raton, FL, 2017.

(32) Vargaftik, N. B. Handbook of Physical Properties of Liquids and Gases. Pure Substances and Mixtures, 2nd ed.; Springer, Berlin, 1975.

(33) Zábranskỳ, M.; Ružička, V., Jr; Domalski, E. S. Heat Capacity of Liquids: Critical Review and Recommended Values. Supplement I. J. Phys. Chem. Ref. Data 2001, 30, 1199-1689.

(34) de Castro, C. A. N.; Li, S. F. Y.; Nagashima, A.; Trengove, R. D.; Wakeham, W. A. Standard Reference Data for the Thermal Conductivity of Liquids. J. Phys. Chem. Ref. Data 1986, 15, 10731086.

(35) Johnson, P. B.; Christy, R. W. Optical Constants of the Noble Metals. Phys. Rev. B 1972, 6, 4370-4379.

(36) Hale, G. M.; Querry, M. R. Optical Constants of Water in the 200-nm to $200-\mu \mathrm{m}$ Wavelength Region. Appl. Opt. 1973, 12, 555563.

(37) Barrat, J. L.; Chiaruttini, F. Kapitza resistance at the liquid-solid interface. Mol. Phys. 2003, 101, 1605-1610.

(38) Ge, Z.; Cahill, D. G.; Braun, P. V. AuPd Metal Nanoparticles as Probes of Nanoscale Thermal Transport in Aqueous Solution. J. Phys. Chem. B 2004, 108, 18870-18875.

(39) Ge, Z. Nanoscale Thermal Transport at Solid-Liquid Interfaces. Ph.D. Thesis, University of Illinois at Urbana-Champaign, 2006.

(40) Shenogina, N.; Godawat, R.; Keblinski, P.; Garde, S. How Wetting and Adhesion Affect Thermal Conductance of a Range of Hydrophobic to Hydrophilic Aqueous Interfaces. Phys. Rev. Lett. 2009, 102, 156101.

(41) Merabia, S.; Shenogin, S.; Joly, L.; Keblinski, P.; Barrat, J. L. Heat transfer from nanoparticles: A corresponding state analysis. Proc. Natl. Acad. Sci. U. S. A. 2009, 106, 15113-15118.

(42) Vo, T. Q.; Kim, B. H. Interface Thermal Resistance between Liquid Water and Various Metallic Surfaces. International Journal of Precision Engineering and Manufacturing 2015, 16, 1341-1346.

(43) Ju, S.; Palpant, B.; Chalopin, Y. Adverse Effects of Polymer Coating on Heat Transport at the Solid-Liquid Interface. J. Phys. Chem. C 2017, 121, 13474-13480. 
(44) Wang, D.; Maize, K.; Song, M.; Boltasseva, A.; Shalaev, V. M.; Shakouri, A.; Kildishev, A. V. Thermoreflectance Imaging of Optically Pumped Gap Plasmon Structures. Proceedings of the Conference on Lasers and Electro-Optics, San Jose, CA, May 13-18, 2018; OSA Publishing, 2018.

(45) Wiesbauer, M.; Wollhofen, R.; Vasic, B.; Schilcher, K.; Jacak, J.; Klar, T. A. Nano-Anchors with Single Protein Capacity Produced with STED Lithography. Nano Lett. 2013, 13, 5672-5678.

(46) Inasawa, S.; Sugiyama, M.; Yamaguchi, Y. Laser-Induced Shape Transformation of Gold Nanoparticles below the Melting Point: The Effect of Surface Melting. J. Phys. Chem. B 2005, 109, 3104-3111.

(47) Fujii, T.; Ishii, A.; Anpo, M. Absorption and fluorescence spectra of rhodamine B molecules encapsulated in silica gel networks and their thermal stability. J. Photochem. Photobiol., A 1990, 54, 231237.

(48) Rhodamine B (C.I. 45170) for microscopy. Safety Data Sheet; Carl Roth GmbH und Co. KG, 2016.

(49) Gu, Q.; Smalley, J. S. T.; Shane, J.; Bondarenko, O.; Fainman, Y. Temperature effects in metal-clad semiconductor nanolasers. Nanophotonics 2015, 4, 26-43.

(50) Prodan, E.; Radloff, C.; Halas, N. J.; Nordlander, P. A Hybridization Model for the Plasmon Response of Complex Nanostructures. Science 2003, 302, 419-422.

(51) Wei, C.; Liu, S. Y.; Zou, C. L.; Liu, Y.; Yao, J.; Zhao, Y. S. Controlled Self-Assembly of Organic Composite Microdisks for Efficient Output Coupling of Whispering-Gallery-Mode Lasers. J. Am. Chem. Soc. 2015, 137, 62-65.

(52) Ta, V. D.; Chen, R.; Sun, H. D. Tuning Whispering Gallery Mode Lasing from Self-Assembled Polymer Droplets. Sci. Rep. 2013, 3,1362 .

(53) Okada, D.; Nakamura, T.; Braam, D.; Dao, T. D.; Ishii, S.; Nagao, T.; Lorke, A.; Nabeshima, T.; Yamamoto, Y. Color-Tunable Resonant Photoluminescence and Cavity-Mediated Multistep Energy Transfer Cascade. ACS Nano 2016, 10, 7058-7063. 